\title{
Power quality improvement of wind turbines using doubly-fed induction machines
}

\author{
C. Saniter, R. Grune, A. Wood and R. Hanitsch
}

C. Saniter (saniter@iee.tu-berlin.de), R. Grune (grune@iee.tu-berlin.de) and R. Hanitsch (prof.hanitsch@iee.tu-berlin.de) are with the Department of Electrical Engineering at the Technical University of Berlin Technical University of Berlin, Sekr. EM 4, Einsteinufer 11, 10587 Berlin, Germany

\begin{abstract}
Alan Wood (a.wood@elec.canterbury.ac.nz) is with the Department of Electrical and Computer Engineering at the University of Canterbury in Christchurch, New Zealand
\end{abstract}

University of Canterbury, Department of Electrical and Computer Engineering, Private Bag 4800, Christchurch, New Zealand

\begin{abstract}
A novel approach to modelling doubly-fed induction machines facing (distorted) ac power networks is presented. It takes place in the frequency-domain and is based on frequency coupling matrices using tensor algebra. Benefits of this comprehensible and fast modelling approach are demonstrated by establishing a feed-forward control signal that reduces stator current harmonics and torque ripple thereby improving the network compatibility and reducing the stress on the mechanical drive train. Dynamic simulations using PSCAD/EMTDC and MATLAB/Simulink are presented demonstrating the effectiveness of the proposed modelling approach and the feed-forward compensation scheme.
\end{abstract}

\section{Key words}

doubly-fed induction machine, harmonics, power quality, feedforward control, simulation

\section{Introduction}

W IND energy applications are becoming increasingly widespread. The installed capacity in Europe increased by $20 \%$ in 2004 to a total installed capacity of 34.205MW. Leading countries were Spain (2065MW), Germany (2037MW) and Great Britain (240MW). Fig. 1 shows the development in the German market [1].

Two main types of wind energy converters are installed almost exclusively; wind energy converters using synchronous machines (with either permanent magnets or separately excited) and doubly-fed induction machines (DFIMs) (see Fig. 2). Both system have high controllability but are also known to generate and to be adversely affected by harmonics [2].

Doubly-fed induction machines have the great advantage that the stator is directly connected to the grid and the rotor-side converter handles a maximum of approximately $30 \%$ of the rated power. Thus, the converters are substantially smaller and cheaper compared to the converters for synchronous machines. However, harmonic and interharmonic interactions with the

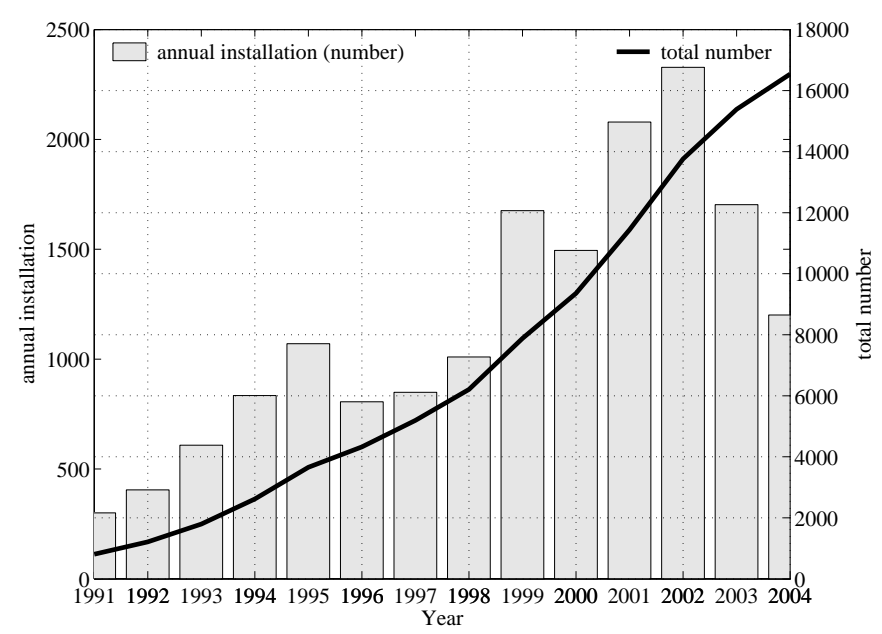

Fig. 1. Number of annual installation and number of total installed wind turbines in Germany

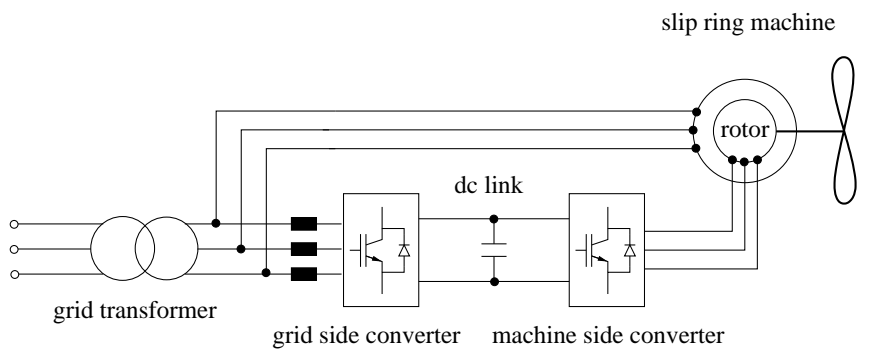

Fig. 2. Wind energy generation concept using doubly-fed induction machines

grid are manifold and have a negative impact on network compatibility. Furthermore, the mechanical stress on the drive train increases. Significant harmonic and interharmonic distortions may result if not properly controlled [3]. For ac systems with unbalanced impedance or with unbalanced voltage sources, controls can be made insensitive to the associated distortion, or can be used to actively reduce distortion levels. One possibility to actively reduce distortion levels has been recently published 
[4]. The strategy is based on knowledge of the ac system distortion, and utilizes feed-forward. The appropriate harmonic rotor voltages that have to be injected in addition to the fundamental rotor voltage are computed for known stator voltage distortions. Knowing the appropriate feed-forward signal is difficult and based on complex computations. A novel approach to modelling doubly-fed induction machines facing distorted ac power networks is presented in this paper. This modelling approach is not only very comprehensible and computationally efficient but also provides an elegant way to obtain the appropriate feed-forward signals including ac network characteristics.

\section{Modelling}

The standard approach to modelling ac induction machines in a rotating reference frame is used. While developing this model, some simplifications of the real system are necessary. These simplifications are commonly made and do not alter the main characteristics. The main assumptions are:

- no saturation of the inductances

- stator and rotor windings are represented through threephase systems

- the distributed windings are sinusoidally distributed along the circumference of the machine, they are represented by concentrated windings

- harmonics caused by the assembly, e.g. slot harmonics, are neglected.

- all spatial quantities (e.g. flux) are spatially sinusoidal

- iron losses and skin effect are negligible

- the stator and rotor resistances are constant

- all rotor quantities are referred to the stator with the appropriate winding ratio

This yields the well-known non-linear state space model in the rotating $d q$-reference frame for induction machines [5]

$$
\begin{aligned}
& \dot{\mathbf{x}}_{\psi}=\mathbf{A} \cdot \mathbf{x}_{\psi}+\mathbf{B} \cdot \mathbf{u} \\
& \mathbf{x}_{\psi}=\left[\psi_{1}^{\mathrm{d}} \psi_{1}^{\mathrm{q}}, i_{2}^{\mathrm{d}}, i_{2}^{\mathrm{q}}\right]^{T} \\
& \dot{\mathbf{x}}_{\psi}=\left[\frac{\mathrm{d} \psi_{1}^{\mathrm{d}}}{\mathrm{d} t}, \frac{\mathrm{d} \psi_{1}^{\mathrm{q}}}{\mathrm{d} t}, \frac{\mathrm{d} i_{2}^{\mathrm{d}}}{\mathrm{d} t}, \frac{\mathrm{d} i_{2}^{\mathrm{q}}}{\mathrm{d} t}\right]^{T} \\
& \mathbf{A}=\left[\begin{array}{cccc}
-\frac{1}{\tau_{1}} & \omega_{\mathrm{dq}} & \frac{L_{\mathrm{h}}}{\tau_{1}} & 0 \\
-\omega_{\mathrm{dq}} & -\frac{1}{\tau_{1}} & 0 & \frac{L_{\mathrm{h}}}{\tau_{1}} \\
\frac{L_{\mathrm{h}}}{\tau_{1} \sigma} & -\omega_{\mathrm{r}} \frac{L_{\mathrm{h}}}{\sigma} & -\frac{L_{1} R_{2} \tau_{1}+L_{\mathrm{h}}^{2}}{\tau_{1} \sigma} & \omega_{2} \\
\omega_{\mathrm{r}} \frac{L_{\mathrm{h}}}{\sigma} & \frac{L_{\mathrm{h}}}{\tau_{1} \sigma} & -\omega_{2} & -\frac{L_{1} R_{2} \tau_{1}+L_{\mathrm{h}}^{2}}{\tau_{1} \sigma}
\end{array}\right] \\
& \mathbf{B}=\left[\begin{array}{cccc}
1 & 0 & 0 & 0 \\
0 & 1 & 0 & 0 \\
-\frac{L_{\mathrm{h}}}{\sigma} & 0 & \frac{L_{1}}{\sigma} & 0 \\
0 & -\frac{L_{\mathrm{h}}}{\sigma} & 0 & \frac{L_{1}}{\sigma}
\end{array}\right] \\
& \mathbf{u}=\left[v_{1}^{\mathrm{d}}, v_{1}^{\mathrm{q}}, v_{2}^{\mathrm{d}}, v_{2}^{\mathrm{q}}\right]^{T}
\end{aligned}
$$

where $v_{1,2}^{\mathrm{d}, \mathrm{q}}$ are the stator and rotor voltages, $\psi_{1}^{\mathrm{d}, \mathrm{q}}$ are the stator flux linkages and $i_{1,2}^{\mathrm{d}, \mathrm{q}}$ are the stator and rotor currents in a rotating $d q$-reference frame. The elements in the state-matrix $\mathbf{A}$ and control matrix $\mathbf{B}$ are the stator and rotor resistances
$R_{1,2}$, the rotor and stator inductances $L_{1,2}=L_{\sigma 1,2}+L_{\mathrm{h}}$ where $L_{\sigma 1,2}$ are the leakage and $L_{\mathrm{h}}$ is the mutual inductance, the rotor and stator time constants $\tau_{1,2}=\frac{L_{1,2}}{R_{1,2}}$ and the modified Blondel's coefficient $\sigma=L_{1} L_{2}-L_{\mathrm{h}}^{2}$. The angular frequency of the electric rotor quantities is $\omega_{2}, \omega_{\mathrm{dq}}$ is the angular frequency of the rotating reference frame and $\omega_{\mathrm{r}}$ is the mechanical speed (in $\frac{\mathrm{rad}}{\mathrm{s}}$ ), scaled with the number of pole-pairs $z_{\mathrm{p}}$. Eqn. (1) fully describes the electrical dynamic behaviour of doubly-fed induction machines. The mechanical behaviour is not included so far. The choice of state variables in Eqn. (1) is not unique. This does not mean that the state of the system at any time is not unique, only that the means of representing the state information is not unique. Any set of state variables will provide the same information about the system. The subscript $\psi$ indicates that the stator fluxes are chosen as states as opposed to the stator currents. The state vector for the latter is accordingly $\left[x_{\mathrm{i}}\right]=\left[i_{1}^{\mathrm{d}}, i_{1}^{\mathrm{q}}, i_{2}^{\mathrm{d}}, i_{2}^{\mathrm{q}}\right]^{T}$ with

$$
\underbrace{\left[\begin{array}{c}
\Delta \psi_{1}^{\mathrm{d}} \\
\Delta \psi_{1}^{\mathrm{q}} \\
\Delta i_{2}^{\mathrm{d}} \\
\Delta i_{2}^{\mathrm{q}} \\
\Delta \omega_{\mathrm{r}}
\end{array}\right]}_{\left[x_{\psi}\right]}=\underbrace{\left[\begin{array}{ccccc}
L_{1} & 0 & L_{\mathrm{h}} & 0 & 0 \\
0 & L_{1} & 0 & L_{\mathrm{h}} & 0 \\
0 & 0 & 1 & 0 & 0 \\
0 & 0 & 0 & 1 & 0 \\
0 & 0 & 0 & 0 & 1
\end{array}\right]}_{\left[L_{\mathrm{i} 2 \psi}\right]} \underbrace{\left[\begin{array}{c}
\Delta i_{1}^{\mathrm{d}} \\
\Delta i_{1}^{\mathrm{q}} \\
\Delta i_{2}^{\mathrm{d}} \\
\Delta i_{2}^{\mathrm{q}} \\
\Delta \omega_{\mathrm{r}}
\end{array}\right]}_{\left[x_{\mathrm{i}}\right]} .
$$

An appropriate non-linear output equation is found by including the equation of motion

$$
\frac{\mathrm{d} \omega_{\mathrm{r}}}{\mathrm{d} t}=\frac{z_{\mathrm{p}}}{\mathrm{J}}\left(T_{\mathrm{el}}-T_{\mathrm{L}}\right)
$$

The torque $T_{\mathrm{L}}$ is the load torque and $T_{\mathrm{el}}$ is the electrical torque developed by the machine

$$
T_{\mathrm{el}}=\frac{3}{2} z_{\mathrm{p}}\left(\psi_{1}^{\mathrm{q}} i_{2}^{\mathrm{d}}-\psi_{1}^{\mathrm{d}} i_{2}^{\mathrm{q}}\right)
$$

For further details see [4], [5]. The following problems are immediately apparent: The output equation is non-linear due to multiplications of state variables and the state matrix is non-linear and time-dependent because of its dependence on the rotor frequency $\omega_{2}$ and the mechanical speed $\omega_{\mathrm{r}}$. For timedomain simulation purposes, however, the state-space Eqn. (1) and the torque Eqn. (4) are fully sufficient and are used in the Simulink model.

\section{Small-signal linearisation}

In order to obtain a linearised model of the machine, the equation of motion is included into the state-space representation. Let the following autonomous set of equations represent the machine's dynamic behaviour

$$
\dot{\mathbf{x}}=\mathbf{f}(\mathbf{x}, \mathbf{u})
$$

where the set of non-linear equations $\mathbf{f}$ relates the state vector $[x(t)]=\dot{\mathbf{x}}$ and the input vector $[u(t)]=\mathbf{u}$ [6]. Taylor series expansion with terms involving second and higher order 
powers of $\Delta x$ and $\Delta u$ neglected yields the linearised state matrix $\left[A_{\Delta}\right]$ and control matrix $\left[B_{\Delta}\right]$ in

$$
\begin{aligned}
& {\left[A_{\Delta}\right]=\left[\begin{array}{ccc}
\left.\frac{\partial f_{1}}{\partial x_{1}}\right|_{\mathbf{x}^{\mathbf{0}}, \mathbf{u}^{\mathbf{0}}} & \cdots & \left.\frac{\partial f_{1}}{\partial x_{5}}\right|_{\mathbf{x}^{\mathbf{0}}, \mathbf{u}^{\mathbf{0}}} \\
\vdots & \ddots & \vdots \\
\left.\frac{\partial f_{5}}{\partial x_{1}}\right|_{\mathbf{x}^{\mathbf{0}}, \mathbf{u}^{\mathbf{0}}} & \cdots & \left.\frac{\partial f_{5}}{\partial x_{5}}\right|_{\mathbf{x}^{\mathbf{0}}, \mathbf{u}^{\mathbf{0}}}
\end{array}\right]} \\
& {\left[B_{\Delta}\right]=\left[\begin{array}{ccc}
\left.\frac{\partial f_{1}}{\partial u_{1}}\right|_{\mathbf{x}^{\mathbf{0}}, \mathbf{u}^{\mathbf{0}}} & \cdots & \left.\frac{\partial f_{1}}{\partial u_{5}}\right|_{\mathbf{x}^{\mathbf{0}}, \mathbf{u}^{\mathbf{0}}} \\
\vdots & \ddots & \vdots \\
\left.\frac{\partial f_{5}}{\partial u_{1}}\right|_{\mathbf{x}^{\mathbf{0}}, \mathbf{u}^{\mathbf{0}}} & \cdots & \left.\frac{\partial f_{5}}{\partial u_{5}}\right|_{\mathbf{x}^{\mathbf{0}}, \mathbf{u}^{\mathbf{0}}}
\end{array}\right]}
\end{aligned}
$$

where $\mathbf{x}^{\mathbf{0}}$ and $\mathbf{u}^{\mathbf{0}}$ describe the steady-state operating or equilibrium point around which the small-signal analysis is carried out. $\Delta$ indicates the small-signal linearised nature of the system. The small-signal linearised state-space representation for the induction machine is given in

$$
\begin{aligned}
& \Delta \dot{\mathbf{x}}_{\psi}=\left[A_{\Delta}\right] \cdot \Delta \mathbf{x}_{\psi}+\left[B_{\Delta}\right] \cdot \Delta \mathbf{u} \\
& \Delta \mathbf{x}_{\psi}=\left[\Delta \psi_{1}^{\mathrm{d}}, \Delta \psi_{1}^{\mathrm{q}}, \Delta i_{2}^{\mathrm{d}}, \Delta i_{2}^{\mathrm{q}}, \Delta \omega_{\mathrm{r}}\right]^{T} \\
& \Delta \dot{\mathbf{x}}_{\psi}=\left[\frac{\mathrm{d} \Delta \psi_{1}^{\mathrm{d}}}{\mathrm{d} t}, \frac{\mathrm{d} \Delta \psi_{1}^{\mathrm{q}}}{\mathrm{d} t}, \frac{\mathrm{d} \Delta i_{2}^{\mathrm{d}}}{\mathrm{d} t}, \frac{\mathrm{d} \Delta i_{2}^{\mathrm{q}}}{\mathrm{d} t}, \frac{\mathrm{d} \Delta \omega_{\mathrm{r}}}{\mathrm{d} t}\right]^{T} \\
& {\left[A_{\Delta}\right]=\left[\begin{array}{ccccc}
-\frac{1}{\tau_{1}} & \omega_{\mathrm{f}} & \frac{L_{\mathrm{h}}}{\tau_{1}} & 0 & 0 \\
-\omega_{\mathrm{f}} & -\frac{1}{\tau_{1}} & 0 & \frac{L_{\mathrm{h}}}{\tau_{1}} & 0 \\
\frac{L_{\mathrm{h}}}{\tau_{1} \sigma} & -\omega_{\mathrm{r}}^{0} \frac{L_{\mathrm{h}}}{\sigma} & a & \omega_{2}^{0} & b \\
\omega_{\mathrm{r}}^{0} \frac{L_{\mathrm{h}}}{\sigma} & \frac{L_{\mathrm{h}}}{\tau_{1} \sigma} & -\omega_{2}^{0} & a & c \\
-d \cdot i_{2}^{\mathrm{q} 0} & d \cdot i_{2}^{\mathrm{d} 0} & d \cdot \psi_{1}^{\mathrm{q} 0} & -d \cdot \psi_{1}^{\mathrm{d} 0} & 0
\end{array}\right]} \\
& a=-\left(\frac{L_{1} R_{2}}{\sigma}+\frac{L_{\mathrm{h}}^{2}}{\tau_{1} \sigma}\right) \\
& b=-\frac{L_{\mathrm{h}}}{\sigma} \psi_{1}^{\mathrm{q} 0}-i_{2}^{\mathrm{q} 0} \\
& c=\frac{L_{\mathrm{h}}}{\sigma} \psi_{1}^{\mathrm{d} 0}+i_{2}^{\mathrm{d} 0} \\
& d=\frac{3}{2} \frac{z_{\mathrm{p}}^{2}}{\mathrm{~J}} \\
& {\left[B_{\Delta}\right]=\left[\begin{array}{ccccc}
1 & 0 & 0 & 0 & 0 \\
0 & 1 & 0 & 0 & 0 \\
-\frac{L_{\mathrm{h}}}{\sigma} & 0 & \frac{L_{1}}{\sigma} & 0 & 0 \\
0 & -\frac{L_{\mathrm{h}}}{\sigma} & 0 & \frac{L_{1}}{\sigma} & 0 \\
0 & 0 & 0 & 0 & -\frac{z_{\mathrm{p}}}{\mathrm{J}}
\end{array}\right]} \\
& \Delta \mathbf{u}=\left[\Delta v_{1}^{\mathrm{d}}, \Delta v_{1}^{\mathrm{q}}, \Delta v_{2}^{\mathrm{d}}, \Delta v_{2}^{\mathrm{q}}, \Delta T_{\mathrm{L}}\right]^{T}
\end{aligned}
$$

Further details on the linearisation process of arbitrary statespace systems can be found in [6]. This small-signal timedomain state-space model does not provide an insight into the generation and propagation of harmonics. It is desirable to represent the system in the frequency-domain, where fundamental characteristics are significantly more apparent. Furthermore, the computation of appropriate rotor voltage injections in the presence of harmonic stator voltage distortions is straightforward and fast.

\section{Small-signal frequency-domain model of the doubly-fed induction machine}

By taking the Laplace transform of Eqn. (8) and by applying Eqn. (2), the state-space representation in the Laplace domain is obtained

$$
s \Delta \mathbf{x}_{\mathrm{i}}(s)-\Delta \mathbf{x}_{\mathrm{i}}(t=0)=\left[A_{\Delta}\right] \Delta \mathbf{x}(s)+\left[B_{\Delta}\right] \Delta \mathbf{u}(s) .
$$

The initial conditions, i.e. the initial distortions, of the system $\Delta \mathbf{x}_{\mathbf{i}}(t=0)$ can be assumed to be zero. Thus, Eqn. (9) now becomes

$$
\underbrace{\left[\begin{array}{c}
\Delta v_{1}^{\mathrm{d}}(s) \\
\Delta v_{1}^{\mathrm{q}}(s) \\
\Delta v_{2}^{\mathrm{d}}(s) \\
\Delta v_{2}^{\mathrm{q}}(s) \\
\Delta T_{\mathrm{L}}(s)
\end{array}\right]}_{\Delta \mathbf{u}(s)}=\underbrace{\left[B_{\Delta}\right]^{-1}\left(s[I]-\left[A_{\Delta}\right]\right)\left[L_{\mathrm{i} 2 \psi}\right]}_{\left[D F_{\Delta}(s)\right]} \underbrace{\left[\begin{array}{c}
\Delta i_{1}^{\mathrm{d}}(s) \\
\Delta i_{1}^{\mathrm{q}}(s) \\
\Delta i_{2}^{\mathrm{d}}(s) \\
\Delta i_{2}^{\mathrm{q}}(s) \\
\Delta \omega_{\mathrm{r}}(s)
\end{array}\right]}_{\Delta \dot{\mathbf{x}}_{\mathrm{i}}(s)}
$$

where $[I]$ is the identity matrix the same size as $\left[A_{\Delta}\right]$. $\left[D F_{\Delta}(s)\right]$ can be expressed explicitly as

$$
\begin{aligned}
& {\left[\begin{array}{ccccc}
L_{1} s+\frac{L_{1}}{\tau_{1}} & -\omega_{\mathrm{dq}} L_{1} & L_{\mathrm{h}} s & -\omega_{\mathrm{dq}} L_{\mathrm{h}} & 0 \\
\omega_{\mathrm{dq}} L_{1} & L_{1} s+\frac{L_{1}}{\tau_{1}} & \omega_{\mathrm{dq}} L_{\mathrm{h}} & L_{\mathrm{h}} s & 0 \\
L_{\mathrm{h}} s & -L_{\mathrm{h}} \omega_{2}^{0} & L_{2} s+R_{2} & -\omega_{2}^{0} L_{2} & \tilde{b} \\
L_{\mathrm{h}} \omega_{2}^{0} & L_{\mathrm{h}} s & \omega_{2}^{0} L_{2} & s L_{2}+R_{2} & \tilde{c} \\
-\tilde{d} \cdot i_{2}^{\mathrm{q} 0} & \tilde{d} \cdot i_{2}^{\mathrm{d} 0} & e & f & -\frac{J}{z_{\mathrm{p}}} s
\end{array}\right]} \\
& \tilde{d}=L_{1} \cdot \frac{3}{2} z_{\mathrm{p}} \\
& \tilde{b}=\frac{L_{\mathrm{h}}}{L_{1}} \psi_{1}^{\mathrm{q} 0}+\frac{\sigma}{L_{1}} i_{2}^{\mathrm{q} 0} \\
& \tilde{c}=-\frac{L_{\mathrm{h}}}{L_{1}} \psi_{1}^{\mathrm{d} 0}-\frac{\sigma}{L_{1}} i_{2}^{\mathrm{d} 0} \\
& e=\frac{3}{2} z_{\mathrm{p}} \psi_{1}^{\mathrm{q} 0}-\frac{3}{2} z_{\mathrm{p}} i_{2}^{\mathrm{q} 0} L_{\mathrm{h}} \\
& f=\frac{3}{2} z_{\mathrm{p}} i_{2}^{\mathrm{d} 0} L_{\mathrm{h}}-\frac{3}{2} z_{\mathrm{p}} \psi_{1}^{\mathrm{d} 0}
\end{aligned}
$$

This paper focuses on the harmonic analysis of doubly-fed induction machines. Thus, the Laplace operator $s=\sigma+\mathrm{j} \omega$ is reduced to $s=\mathrm{j} \omega$. Most elements in $\left[D F_{\Delta}(\mathrm{j} \omega)\right]$ are constant and not frequency dependent. In order to avoid complex computations and in order to obtain a fast and comprehensible model for the doubly-fed induction machine, the transfer matrix $\left[D F_{\Delta}(\mathrm{j} \omega)\right]$ is computed for $n$ frequency components, spaced $\Delta \omega$ apart. The use of complex numbers is avoided by separating the real and imaginary components and applying tensor algebra. For details on this modelling approach see [7], [8]. The resulting $n$ transfer matrices $\left[D F_{\Delta}(\Delta \omega)_{n}\right]$ are merged into one matrix. This matrix is called frequency coupling matrix $D F_{\Delta}$ and is given as

$$
\left[\begin{array}{c}
\Delta v_{1}^{\mathrm{d}} \\
\Delta v_{1}^{\mathrm{q}} \\
\Delta v_{2}^{\mathrm{d}} \\
\Delta v_{2}^{\mathrm{q}} \\
\Delta T_{\mathrm{L}}
\end{array}\right]=D F_{\Delta}\left[\begin{array}{c}
\Delta i_{1}^{\mathrm{d}} \\
\Delta i_{1}^{\mathrm{q}} \\
\Delta i_{2}^{\mathrm{d}} \\
\Delta i_{2}^{\mathrm{q}} \\
\Delta \omega_{\mathrm{r}}
\end{array}\right]
$$

$D F_{\Delta}=\left[\begin{array}{ccccc}D F_{\Delta 11} & D F_{\Delta 12} & D F_{\Delta 13} & D F_{\Delta 14} & 0 \\ D F_{\Delta 21} & D F_{\Delta 22} & D F_{\Delta 23} & D F_{\Delta 24} & 0 \\ D F_{\Delta 31} & D F_{\Delta 32} & D F_{\Delta 33} & D F_{\Delta 34} & D F_{\Delta 35} \\ D F_{\Delta 41} & D F_{\Delta 42} & D F_{\Delta 43} & D F_{\Delta 44} & D F_{\Delta 45} \\ D F_{\Delta 51} & D F_{\Delta 52} & D F_{\Delta 53} & D F_{\Delta 54} & D F_{\Delta 55}\end{array}\right]$ 
All sub-matrices $D F_{* *}$ are matrices in the appropriate tensor representation and contain only real numbers. The frequency and frequency cross-coupling information is embedded in the coefficients that are building the frequency coupling matrix. Fig. 3 is a visualisation of the matrix structure of $D F_{\Delta}$. The diagonal line in each sub-matrix represents the location of tensor elements in the matrix.

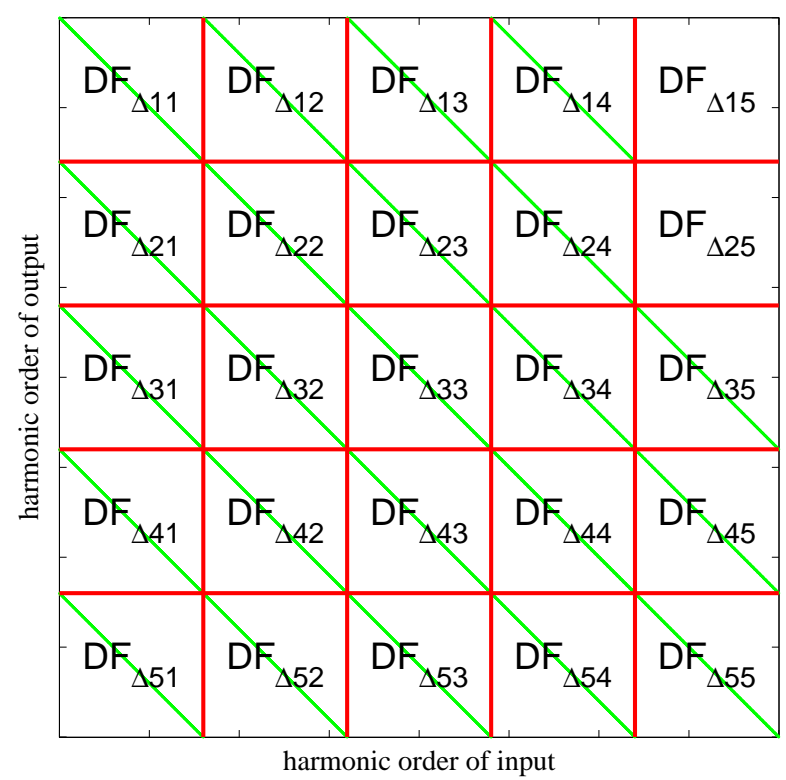

Fig. 3. Structure of the frequency coupling matrix $D F_{\Delta}$ of the linearised doubly-fed induction machine

The sparse nature of the matrix is immediately apparent. This indicates very little frequency cross-coupling but also clearly shows all resultant frequencies for any harmonic perturbation. In its present form this frequency coupling matrix has a drawback. Rotor and stator quantities are transformed into the same rotating reference frame, thus information about the real frequencies and their associated frequency shifts from the rotor into the stator and vice versa is lost. This information can be retrieved by transforming the machine model from the $d q$-reference frame to sequence components. This is accomplished in the time-domain by applying Eqn. (15). A similar expression exists in the frequency-domain and can be found in [8].

Rearranging Eqn. (12) yields

$$
\begin{aligned}
{\left[\begin{array}{c}
\Delta v_{1}^{\mathrm{d}} \\
\Delta v_{1}^{\mathrm{q}} \\
\Delta T_{\mathrm{L}} \\
\Delta v_{2}^{\mathrm{d}} \\
\Delta v_{2}^{\mathrm{q}}
\end{array}\right]=\left[\begin{array}{ll}
A_{11} & A_{12} \\
A_{21} & A_{22}
\end{array}\right]\left[\begin{array}{c}
\Delta i_{1}^{\mathrm{d}} \\
\Delta i_{1}^{\mathrm{q}} \\
\Delta \omega_{\mathrm{r}} \\
\Delta i_{2}^{\mathrm{d}} \\
\Delta i_{2}^{\mathrm{q}}
\end{array}\right] } \\
{\left[A_{11}\right]=\left[\begin{array}{cc}
D F_{\Delta 11} & D F_{\Delta 12} \\
D F_{\Delta 21} & D F_{\Delta 22} \\
D F_{\Delta 51} & D F_{\Delta 52}
\end{array}\right] } \\
{\left[A_{12}\right]=\left[\begin{array}{lll}
D F_{\Delta 13} & D F_{\Delta 14} & 0 \\
D F_{\Delta 23} & D F_{\Delta 24} & 0 \\
D F_{\Delta 53} & D F_{\Delta 54} & D F_{\Delta 55}
\end{array}\right] }
\end{aligned}
$$

$$
\begin{aligned}
{\left[A_{21}\right] } & =\left[\begin{array}{ll}
D F_{\Delta 41} & D F_{\Delta 42} \\
D F_{\Delta 31} & D F_{\Delta 32}
\end{array}\right] \\
{\left[A_{22}\right] } & =\left[\begin{array}{lll}
D F_{\Delta 43} & D F_{\Delta 44} & D F_{\Delta 45} \\
D F_{\Delta 33} & D F_{\Delta 34} & D F_{\Delta 35}
\end{array}\right]
\end{aligned}
$$

which can finally be expressed as

$$
\left[\begin{array}{c}
\Delta i_{2}^{\mathrm{d}} \\
\Delta i_{2}^{\mathrm{q}} \\
\Delta \omega_{\mathrm{r}} \\
\Delta v_{2}^{\mathrm{d}} \\
\Delta v_{2}^{\mathrm{q}}
\end{array}\right]=\left[\begin{array}{cc}
-A_{12}^{-1} A_{11} & A_{12}^{-1} \\
A_{21}-A_{22} A_{21}^{-1} A_{11} & A_{22} A_{12}^{-1}
\end{array}\right]\left[\begin{array}{c}
\Delta i_{1}^{\mathrm{d}} \\
\Delta i_{1}^{\mathrm{q}} \\
\Delta v_{1}^{\mathrm{d}} \\
\Delta v_{1}^{\mathrm{q}} \\
\Delta T_{\mathrm{L}}
\end{array}\right] .
$$

This allows the computation of the rotor voltages to be injected for a given and known stator voltage distortion and desired stator current harmonics. It is neither sensible nor realistic that the load torque variations could be chosen freely. They are imposed onto the system by the wind turbine. Thus, solving Eqn. (14) with $\Delta i_{1}^{\mathrm{d}, \mathrm{q}}=0$ and $\Delta T_{\mathrm{L}}=0$ yields the desired additional rotor voltages $\Delta v_{2}^{\mathrm{d}, \mathrm{q}}$ for given and known stator voltage distortions $\Delta v_{1}^{\mathrm{d}, \mathrm{q}}$.

\section{Results}

Dynamic simulations using PSCAD/EMTDC and MATLAB/Simulink are used in order to verify the proposed feedforward control approach. Table I summarises the machine parameters for this simulation.

TABLE I

SIMULATION PARAMETERS FOR A GRID-CONNECTED DOUBLY-FED INDUCTION MACHINE [9]

\begin{tabular}{|c|c|}
\hline stator base frequency & $f_{1}=50 \mathrm{~Hz}$ \\
\hline stator phase rms voltage & $v_{1}=230 \mathrm{~V}$ \\
\hline stator resistance & $R_{1}=1.05 \Omega$ \\
\hline rotor resistance & $R_{2}=1.315 \Omega$ \\
\hline mutual inductance & $L_{\mathrm{h}}=0.16775 \mathrm{H}$ \\
\hline stator leakage inductance & $L_{\sigma 1}=7.99 \mathrm{mH}$ \\
\hline rotor leakage inductance & $L_{\sigma 2}=12.96 \mathrm{mH}$ \\
\hline number of pole pairs & $z_{\mathrm{p}}=2$ \\
\hline inertia & $J=0.0235 \mathrm{Nms}^{2}$ \\
\hline ratio of turns & $N=1$ \\
\hline
\end{tabular}

Fig. 4 shows the circuit which is used in PSCAD/EMTDC and MATLAB/Simulink to analyse the harmonic response of the doubly-fed induction machine and the proposed feed-forward control approach.

The undistorted three-phase grid voltage $v_{1}^{\prime}$ and a $5^{\text {th }}$ harmonic negative sequence distortion $\Delta v_{1}^{\prime}=9.8 \mathrm{~V}$ at $3 \%$ of the fundamental voltage level are supplied to the stator terminals. The load torque is $T_{\mathrm{L}}=1.4$ (p.u.) $=51 \mathrm{Nm}$. The resultant rotational speed consists of two components; the operating point speed $\omega_{\mathrm{r}}$ and an oscillating component $\Delta \omega_{\mathrm{r}}$. The rotor voltage source $\Delta v_{2}$ is controlled in such a way as to inject the appropriate rotor voltages computed by solving Eqn. (14) 


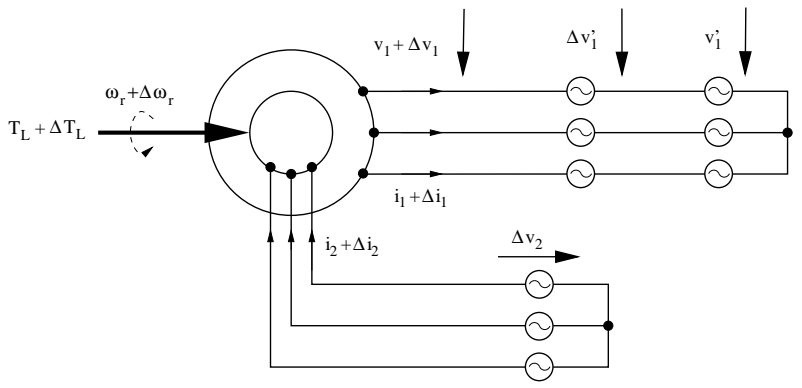

Fig. 4. Simulation circuit in PSCAD/EMTDC for the doubly-fed induction machine

and the inverse Park's transformation in Eqn. (15)

$\left[\begin{array}{c}v_{2}^{\mathrm{a}} \\ v_{2}^{\mathrm{b}} \\ v_{2}^{\mathrm{c}}\end{array}\right]=\left[\begin{array}{cc}\sin (\omega t+\varphi) & \cos (\omega t+\varphi) \\ \sin \left(\omega t+\varphi-\frac{2 \pi}{3}\right) & \cos \left(\omega t+\varphi-\frac{2 \pi}{3}\right) \\ \sin \left(\omega t+\varphi+\frac{2 \pi}{3}\right) & \cos \left(\omega t+\varphi+\frac{2 \pi}{3}\right)\end{array}\right]\left[\begin{array}{c}v_{2}^{\mathrm{d}} \\ v_{2}^{\mathrm{q}}\end{array}\right]$

Fig. 5 shows the positive and negative sequence frequency components in the stator current for a $5^{\text {th }}$ harmonic negative sequence stator voltage distortion at $3 \%$ of the fundamental voltage level. The results are calculated using MATLAB/Simulink. Fig. 6 shows the same, this time for results obtained with PSCAD/EMTDC.
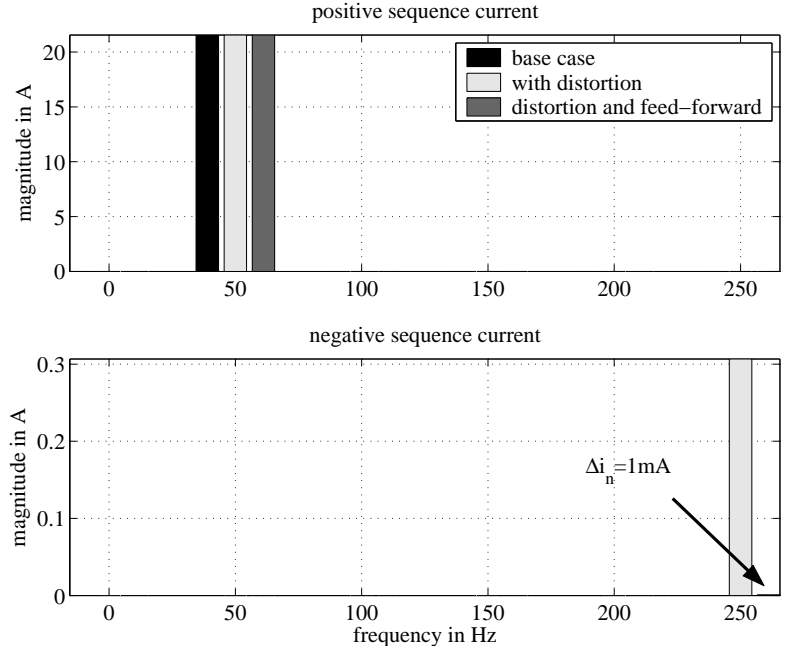

Fig. 5. Stator current spectra for undistorted operation, distorted operation and distorted operation with feed-forward control, $T_{\mathrm{L}}=1.4$ (p.u.), Simulink

Both simulations produce identical results for the base case and distorted case. It can be seen that the $5^{\text {th }}$ harmonic negative sequence current is relatively small at approx. $1.5 \%$ of the fundamental current level. Furthermore, both simulations show that the proposed feed-forward control approach is very effective. The positive sequence, or fundamental current component at $50 \mathrm{~Hz}$, is not affected. Results obtained with MATLAB/Simulink show an almost perfect cancellation of the $5^{\text {th }}$ harmonic negative sequence current, whereas the results obtained with PSCAD/EMTDC are not quite as good. The reason for this lies within the simulation methodology. The
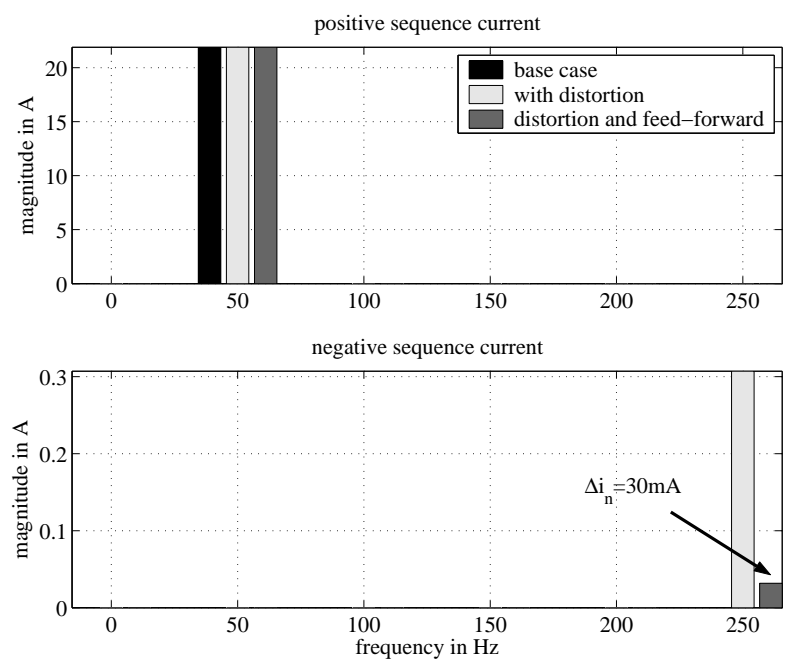

Fig. 6. Stator current spectra for undistorted operation, distorted operation and distorted operation with feed-forward control, $T_{\mathrm{L}}=1.4$ (p.u.), PSCAD/EMTDC

PSCAD/EMTDC results are affected by numerical inaccuracies that are inherent to the simulation program. While it is possible to synchronise the stator and rotor voltages in Simulink, this is not possible in PSCAD/EMTDC resulting in a slow drift of the sinusoidally varying voltages. In either case the $5^{\text {th }}$ harmonic current is reduced substantially from its uncorrected value of approx. $\Delta i_{\mathrm{n}}=305 \mathrm{~mA}$.

Fig. 7 shows the torque frequency spectra obtained with MATLAB/Simulink and Fig. 8 shows the torque frequency spectra obtained with PSCAD/EMTDC.

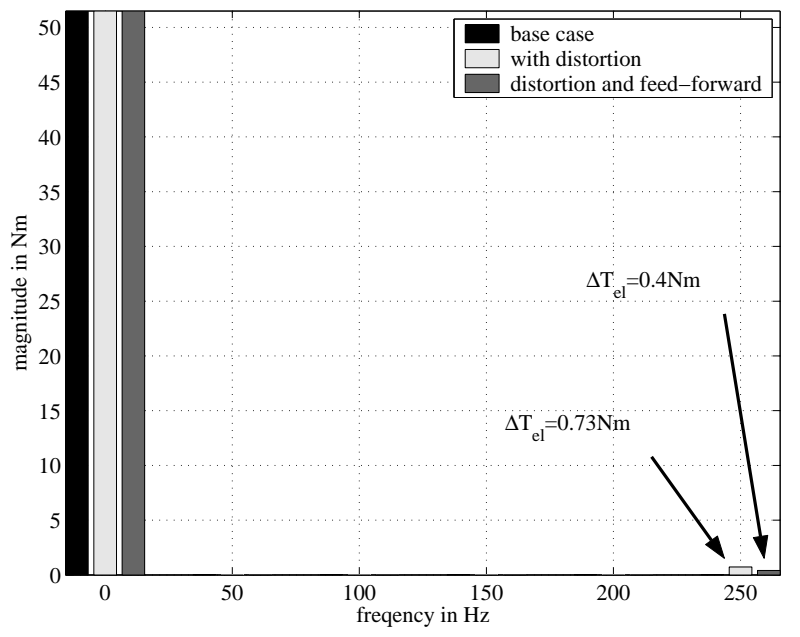

Fig. 7. Torque frequency spectra, Simulink

Both simulations show almost identical results. It can be seen that $\mathrm{a} 6^{\text {th }}$ harmonic torque ripple results from $5^{\text {th }}$ harmonic negative sequence stator currents. The torque ripple is relatively small at approx. $1.5 \%$ of the fundamental level. However, it still has a detrimental impact on the drive train. It can be seen that the torque ripple is reduced even though the feed-forward voltages are computed for zero harmonic currents 


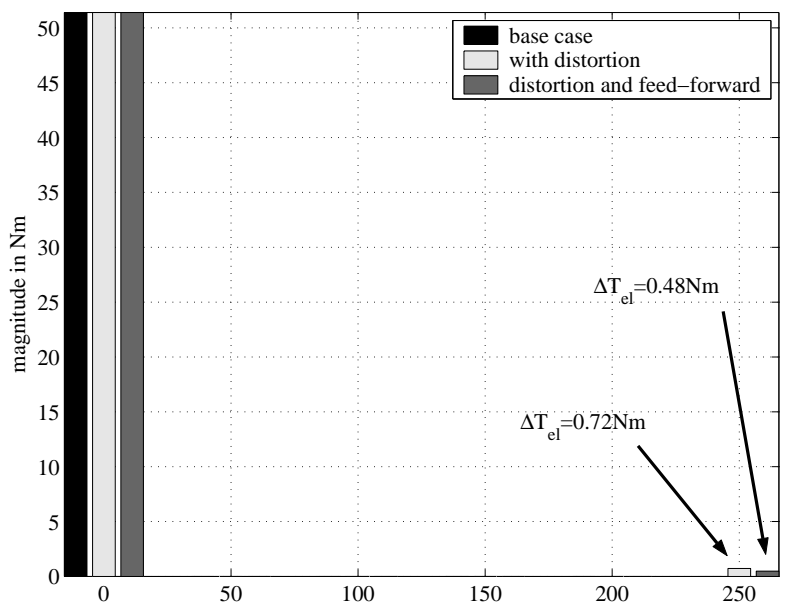

Fig. 8. Torque frequency spectra, PSCAD/EMTDC

not for zero torque ripple. A method is presented in [4] that leads to near zero torque ripple.

Finally, rotor voltages as previously computed, with unchanged magnitude and phase angle, are injected into the rotor but the load torque is reduced to zero $T_{\mathrm{L}}=0$. The resulting frequency spectra for the positive and negative sequence stator currents are shown in Fig. 9.
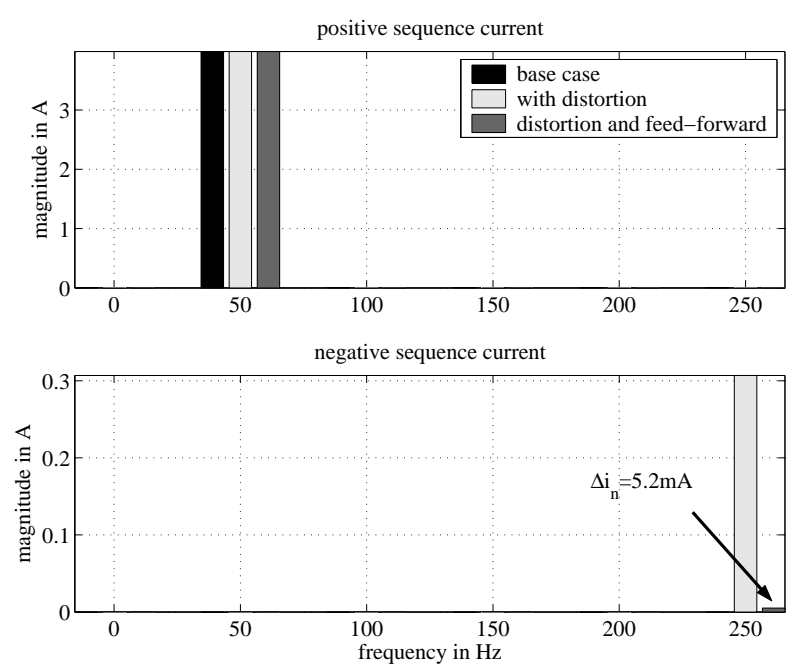

Fig. 9. Stator current spectra for undistorted operation, distorted operation and distorted operation with feed-forward control, $T_{\mathrm{L}}=0$, Simulink

The frequency of the rotor voltages has to be adapted to the new operating point conditions. If the frequency remained unchanged, significant additional distortions result, since the induced emf due to the additional rotor voltages and the harmonic stator voltage would then oscillate at different frequencies. It can be seen that the $5^{\text {th }}$ harmonic stator current remains unchanged compared to the previous test case. It is relatively large at approx. $8 \%$ of the fundamental current level without feed-forward control. The compensation is still very effective at no-load even though the compensation voltages are calculated for a given operating point $\left(T_{\mathrm{L}}=1.4\right.$ (p.u. $\left.)\right)$ and the new operating point differs significantly at $T_{\mathrm{L}}=0$. Thus, the linearisation is truly reasonable and the obtained model covers a large operating range.

\section{Discussion}

It can be clearly seen from Fig. 5 to Fig. 9 that the compensation scheme is effective and significantly reduces the harmonic current flow and torque ripple due to stator voltage harmonics. The reduction in the distortion level of doublyfed induction machines can be explained easily if the stator resistance is ignored. The stator voltage and flux linkage are coupled through the law of induction

$$
\Delta v_{1}^{\mathrm{d}, \mathrm{q}}=\frac{\mathrm{d} \Delta \psi_{1}^{\mathrm{d}, \mathrm{q}}}{\mathrm{d} t}
$$

The stator flux linkage is given through

$$
\Delta \psi_{1}^{\mathrm{d}, \mathrm{q}}=L_{1} \Delta i_{1}^{\mathrm{d}, \mathrm{q}}+L_{\mathrm{h}} \Delta i_{2}^{\mathrm{d}, \mathrm{q}} .
$$

Thus, the rotor current has to provide the change in the stator flux

$$
\Delta i_{2}^{\mathrm{d}, \mathrm{q}}=\frac{1}{L_{\mathrm{h}}} \Delta \psi_{1}^{\mathrm{d}, \mathrm{q}}
$$

for harmonic stator currents $\Delta i_{1}^{\mathrm{d}, \mathrm{q}}=0$. The rotor current can be computed solving the last two lines of Eqn. (1)

$$
\begin{aligned}
\frac{\mathrm{d} i_{2}^{\mathrm{d}}}{\mathrm{d} t} & =\frac{L_{\mathrm{h}}}{\tau_{1} \sigma} \psi_{1}^{\mathrm{d}}-\omega_{\mathrm{r}} \frac{L_{\mathrm{h}}}{\sigma} \psi_{1}^{\mathrm{q}}+a i_{2}^{\mathrm{d}}+\omega_{2} i_{2}^{\mathrm{q}}-\frac{L_{\mathrm{h}}}{\sigma} v_{1}^{\mathrm{d}}+\frac{L_{1}}{\sigma} v_{2}^{\mathrm{d}} \\
\frac{\mathrm{d} i_{2}^{\mathrm{q}}}{\mathrm{d} t} & =\omega_{\mathrm{r}} \frac{L_{\mathrm{h}}}{\sigma} \psi_{1}^{\mathrm{d}}+\frac{L_{\mathrm{h}}}{\tau_{1} \sigma} \psi_{1}^{\mathrm{q}}-\omega_{2} i_{2}^{\mathrm{d}}+a i_{2}^{\mathrm{q}}-\frac{L_{\mathrm{h}}}{\sigma} v_{1}^{\mathrm{q}}+\frac{L_{1}}{\sigma} v_{2}^{\mathrm{q}}
\end{aligned}
$$

Solving Eqn. (14) could be considered a "short-cut" to the above explanation.

There are some constraints on this type of control. Firstly, the stator voltage distortion has to be known in advance. As most grid voltage distortions can be expected to change very slowly the necessary signal processing can be conducted slowly. Secondly, this feed-forward control approach is intended to be a steady state frequency specific harmonic cancellation and should consequently be made insensitive to transients. Thirdly, the calculation of the injected rotor voltages relies on a linearised model. The model is linearised around a certain base case operating point. Base case operating point conditions are embedded in the resulting transfers. As a consequence, the rotor voltage injections have to be re-calculated for every new operating point or left unchanged for a slightly less effective cancellation.

\section{Conclusions}

The transfers of the doubly-fed induction are derived for a given operating point around which the non-linear set of equations is linearised. The resulting transfers are subsequently transformed into the frequency-domain and represented through frequency coupling matrices using tensors. The benefits of representing the doubly-fed induction machine 
through frequency coupling matrices are twofold: firstly, the modelling approach uses only linear algebra. Thus it is extremely fast. Secondly, it is very comprehensible in terms of harmonic generation and propagation. The latter provides an excellent insight into the mechanisms through which harmonics are transformed and generated. This was used to implement a feed-forward control scheme that significantly reduces stator current harmonics and torque oscillations in the presence of stator voltage distortions. Appropriate rotor voltage injections can be easily computed if the stator voltage contains more than one harmonic component, since the feed-forward control strategy is based on a linear model. The reduction of stator current harmonics improves the network compatibility. The reduction of electric torque pulsations reduces the mechanical stress of the drive train. Both are particularly important for wind power applications that are likely to be connected to the public grid at the medium voltage level, and where the gear-box is the component which is most likely to fail.

\section{References}

1) Bundesverband Windenergie, "Zahlen und Fakten", 2004. http://www.wind-energie.de/index.php?id=287

2) D. Schulz, "Untersuchungen von Netzrückwirkungen durch netzgekoppelte Photovoltaik- und Windkraftanlagen”, PhD thesis, TU Berlin, 2002.

3) C. Saniter, D. Schulz and R. Hanitsch, "Harmonics and interharmonics generated by wind energy converters - measurements and a novel modelling approach in the frequency domain", in Proceedings of the ELTRA Workshop, (Billund), 2003.

4) R. Grune, "Interfacing doubly-fed induction machine generators to disturbed ac power networks", Diplomarbeit, TU Berlin, 2005.

5) C. Ong, "Dynamic Simulation of Electric Machinery", Prentice/Hall International, Inc., 1. ed., 1998.

6) P. Kundur, "Power system stability and control", McGraw-Hill, 1994.

7) C. Saniter, R. Hanitsch, C. Osauskas and H. Laird, “A small-signal frequency domain model of a controlled PWM converter", IEEE Postgraduate Power Conference, (Budapest), IEEE, 2002.

8) A. Wood and C. Osauskas, "A linear frequency-domain model of a STATCOM", Transactions on Power Delivery, vol. 19, pp. 1410 - 1418, IEEE, 2004.

9) F. Milde, "Simulation des Maschinenverhaltens mit TUTSIM”, VDE Verlag, 1. ed., 1990. 